\title{
Research Paper: A New Radiographic Method for Estimating Tibial Malrotation
}

\author{
Hamidreza Yazdi $^{1}$ (D, Mohammad Taher Ghaderi ${ }^{\text {** }}$ (D), Alireza Yousof Gomrokchi ${ }^{1}$ Parham Pezeshk $^{1}$ \\ 1. Bone and Joint Reconstruction Research Center, Shafa Orthopedic Hospital, Iran University of Medical Sciences, Tehran, Iran
}

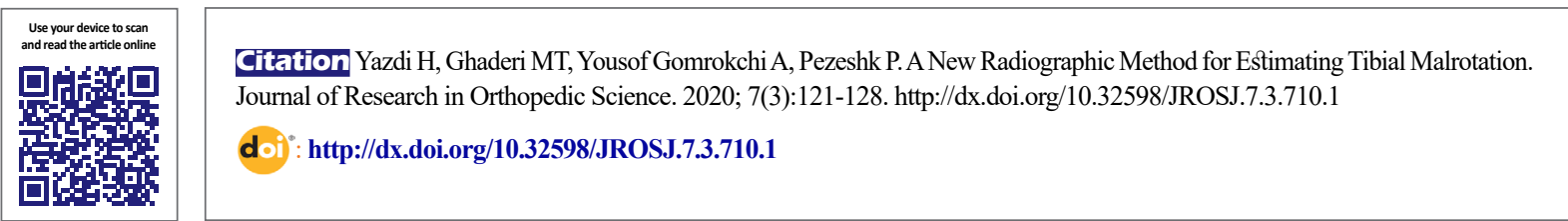

\section{(c) (i) (3)}

Article info:

Received: 26 Jun 2020

Revised: 30 Jun 2020

Accepted: 10 Jul 2020

Available Online: 01 Aug 2020

Keywords:

Radiographic method, Tibial rotation, Tibial malrotation, Tibial fracture fixation

\begin{abstract}
A B S T RA C T
Background: Diaphyseal tibial fractures are the most frequent bone fractures in the body and are usually treated with intramedullary nailing method. However, this approach is responsible for $41 \%$ of the rotational deviation.

Objectives: This study aimed to provide a radiographic evaluation method to determine tibial malrotation in closed fixation of tibia bone fractures during or after the operation.

Methods: This study was conducted in a university hospital from May 2015 to March 2016. All patients referring to the hospital with the complaints of minor trauma around the ankle and knee requiring radiographic evaluation of both joints were enrolled in the study. The inclusion criteria included being 20 and 50 years old; having normal axial, sagittal, and coronal lower limb alignment; lacking previous lower limb injury (such as fractures of the tibia or fibula), ankle or knee sprain; not having previous lower limb surgery, metabolic or congenital bone diseases, or malignancy. In all cases, a standard Anteroposterior (AP) radiograph of the knee was taken, and then, without changing the limb position or image setting, an AP radiograph of the ankle was obtained. The overlap between the distal tibia and fibula was measured in the PACS program environment.

Results: Fifty cases were included in this study. The Mean \pm SD ages of males and females were $29.08 \pm 2.49$ years and $31.46 \pm 2.04$ years, respectively. The range of distal tibia-fibula overlap one centimeter above the tibiotalar joint line was 7.81 to $9.09 \mathrm{~mm}$ (confidence interval of 95\%), and its percentage to the fibula shaft width at the same level was $49.43 \%$ to $54.35 \%$.

Conclusion: According to the results, distal tibia-fibula overlap when the knee is in the true AP position, regardless of the side and gender, is 7.81 to $9.09 \mathrm{~mm}$ or $49.43 \%$ to $54.35 \%$.
\end{abstract}

\section{Introduction}

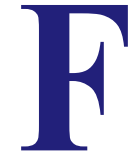

or many years, the tibial diaphyseal fracture has been the most common long-bone fracture in the body (approximately $80 \%$ of the fractures) [1]. Surgery is the most common method of treating displaced fractures in tibial diaphysis which can be carried out through reduction with direct or indirect techniques. In the indirect reduction technique, the fracture site is not opened.

\section{* Corresponding Author:}

Mohammad Taher Ghaderi, MD.

Address: Bone and Joint Reconstruction Research Center, Shafa Orthopedic Hospital, Iran University of Medical Sciences, Tehran, Iran.

Phone: +98 (21) 22128120

E-mail: dr.ghaderim@yahoo.com 
This technique has many advantages over the direct method, such as an increased chance of bone healing and decreased chance of infection. With this method, bone fixation can be done with a plate or a nail [1].

Previous studies have reported that the best surgical treatment method for tibia fracture is the closed fixation with intramedullary nailing [1]. The disadvantages of this procedure are difficulty in its performance and malalignment in sagittal, coronal, and axial planes. Recent studies have indicated that $41 \%$ of rotational malalignment occurs in this type of fixation [1]. Rotational malalignment can be evaluated with various clinical and radiographic methods such as Thigh-Foot Angle (TFA) [2], Transmalleolar Thigh Angle (TTA) [3], radiographic evaluation [3], ultrasonography [4], fluoroscopy [5, 6], and CT scan [7-9].

According to traditional beliefs, the smallest rotational malalignment will result in a clear decline in function, patellofemoral instability [10], disturbance in walking [11], changes in the biomechanics of the knee and ankle joints in the long term, and eventually, early destruction of these joints [12-16] which may lead to the need for corrective surgery, especially in symptomatic cases [1].

Compared to the tibia in the proximal joint, the tibial articular surface in the distal region has been rotated about 20 degrees (4.3 to 39.5 degrees), which is called the normal tibial rotation [1].

The fibula bone overlaps the tibia proximally and distally. Distal tibia-fibula overlap is the maximum extent of the distal fibula and anterior tibia tubercle within 10 $\mathrm{mm}$ of the ankle articular line [17]. The range of the tibia-fibula overlap in anteroposterior radiography is 7.3 to $7.7 \mathrm{~mm}$ in females and 7.9 to $9 \mathrm{~mm}$ in males. Generally, it is 8.1 to $8.5 \mathrm{~mm} \mathrm{[18]} \mathrm{that} \mathrm{changes} \mathrm{from} 10.8 \mathrm{~mm}$ to $2.3 \mathrm{~mm}$ when the ankle rotates 5 degrees outward or 25 degrees inward [19]. It seems that the extent of the tibia-fibula distal overlap would be different in the AP radiography of an ankle with rotation in the distal part when the proximal segment is fixed.

Among clinical and radiographic methods, CT scan is the most accurate technique for evaluating the distal rotation of the tibia [20]. However, CT scan is not recommended routinely because of the high dose of radiation associated with it and its less availability [17]. Clinical methods cannot be used during surgery because of the patient's position. Besides, External Tibial Torsion (ETT) and Internal Tibial Torsion (ITT) are reduced in clinical evaluations up to 22 and 31 degrees, respectively [21]. Therefore, it is necessary to find an easy and accessible method, especially in the operating room, to evaluate tibial torsion in suspicious cases.

The current study measured distal tibia-fibula overlap in cases with normal lower limb alignment with the knee in the true Anteroposterior (AP) position to provide a method for determining tibial rotational malalignment during or after the operation.

\section{Methods}

Patient recruitment was conducted in a university hospital from May 2015 to March 2016. All patients referring to the hospital with complaints of minor trauma around the ankle and knee which needed radiographic evaluation of both joints were enrolled in the study.

The inclusion criteria were the subjects of 20 to 50 years old with no history of major lower limb injury, such as a fracture or sprain, and no congenital or metabolic diseases. The selected individuals had no clear deformity in the coronal, sagittal, or axial planes. The exclusion criteria included any gross coronal, sagittal, or axial deformities upon clinical examination or a history of lower limb fracture or surgery. This research has been approved by the IRB of the authors' affiliated institutions.

During image acquisition, the knee was securely fixed in a true AP position. To evaluate any coronal malalignment, one metal rod was placed across the weight-bearing line, and its position to the medial tibial eminence was evaluated on the radiograph. Radiographs were then taken with the knee in a true AP position, a distance of $100 \mathrm{~cm}$ from the radiographic bed, 3 to 5 degrees of internal rotation in the knee, $60 \mathrm{kV}$ dose of radiation, and the center of the rays pointed at $1.5 \mathrm{~cm}$ distal to the patellar distal pole [22]. All images were evaluated to ensure a true AP position with the metal rod in the correct position (Figure 1).

Then a radiograph of the ankle was taken with no change in image settings or limb positioning. The tibiafibula distal overlap was measured in the PACS program environment. For this purpose, a line was drawn parallel to the distal tibial articular surface at a distance of $10 \mathrm{~mm}$ from the joint line. Then, the tibia-fibula overlap was measured at this level. The overlap percentage was measured by dividing the length of the tibia-fibula overlap by the fibula width at the same level (Figure 2). All measurements were done twice by two different authors, and the mean measurement was calculated. The magnification percentage was considered for all cases. 


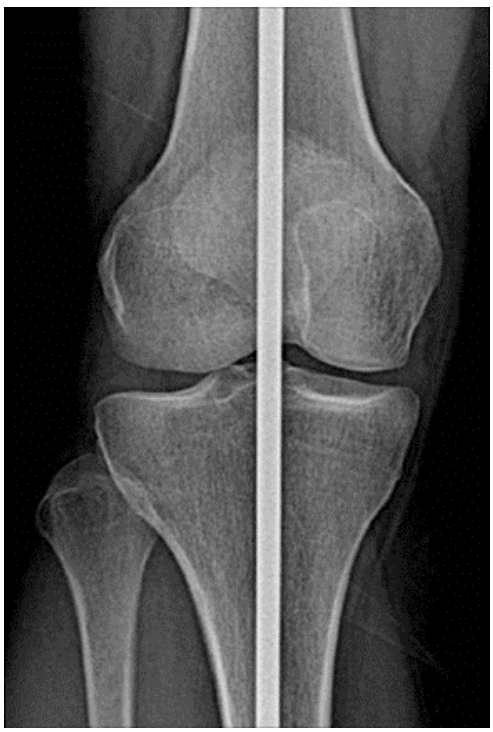

Figure 1. True anteroposterior radiograph of the knee

Orthopedic Science

\section{Results}

Fifty-four patients were enrolled in this study. Four patients were excluded due to abnormal tibial rotation (thigh-foot angle $>30$ degrees).

Fifty patients ( 25 males and 25 females) were evaluated. The Mean $\pm \mathrm{SD}$ age of males was $29.08 \pm 2.49$ years (range: 26.56-31.57 years) and of females 31.46 \pm 2.04 years (31.57- 29.42 years).

In females, the distal tibia-fibula overlap values in the right and left ankles were 6.69 to $8.33 \mathrm{~mm}$ and 6.66 to $8.3 \mathrm{~mm}$, respectively. In males, they were 8.54 to 10.46 $\mathrm{mm}$ and 8.50 to $10.38 \mathrm{~mm}$ in the right and left ankles, respectively. Statistical analysis showed no significant difference in measurements between right and left ankles in males and females $(\mathrm{P}=0.45)$. Regardless of the side, the mean distal tibia-fibula overlap in males and females was 8.52 to $10.42 \mathrm{~mm}$ and 6.68 to $8.32 \mathrm{~mm}$, respectively.

Statistical analysis also showed no significant differences in overlap measurements between males and females $(\mathrm{P}=0.78)$. Regardless of gender or side, the range of the distal tibia-fibula overlap was 7.81 to $9.09 \mathrm{~mm}(95 \% \mathrm{CI})$ (Figure 3).

In females, the percentage of tibia-fibula distal overlap at $1 \mathrm{~cm}$ above the distal tibial articular surface ranged from $46.51 \%$ to $54.43 \%$ with a $95 \%$ CI in the left ankle and $46.82 \%$ to $54.46 \%$ with a $95 \%$ CI in the right ankle.

In males, the percentage of tibia-fibula distal overlap at $1 \mathrm{~cm}$ from the joint surface ranged from $50.07 \%$ to

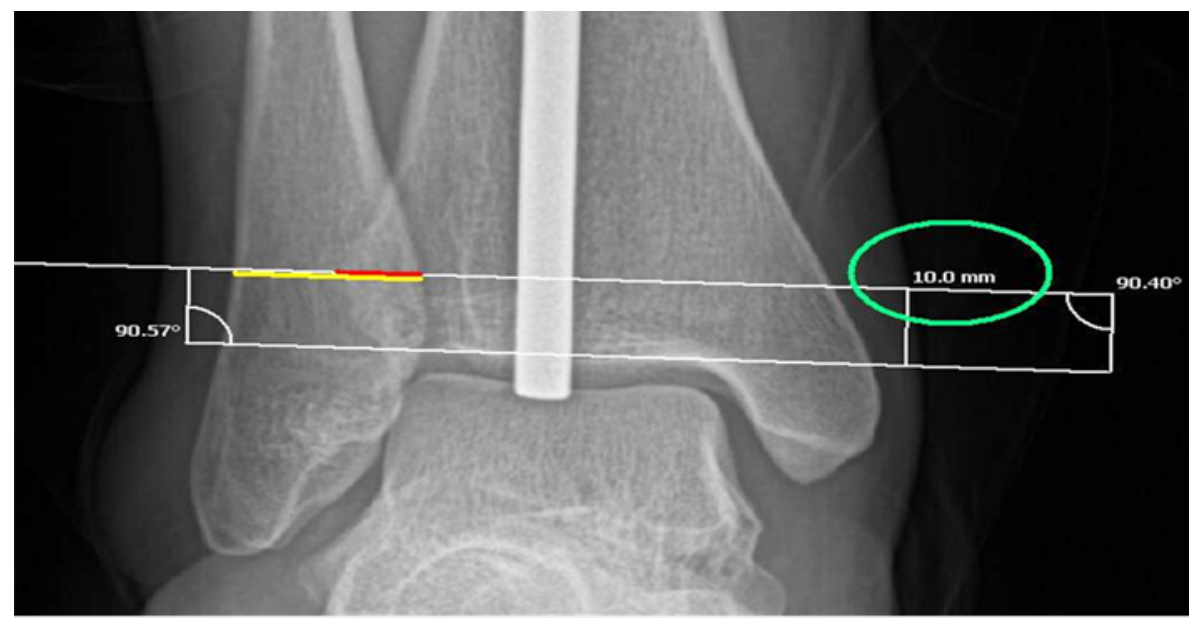

Figure 2. Distal tibia-fibula overlapping measurement method 


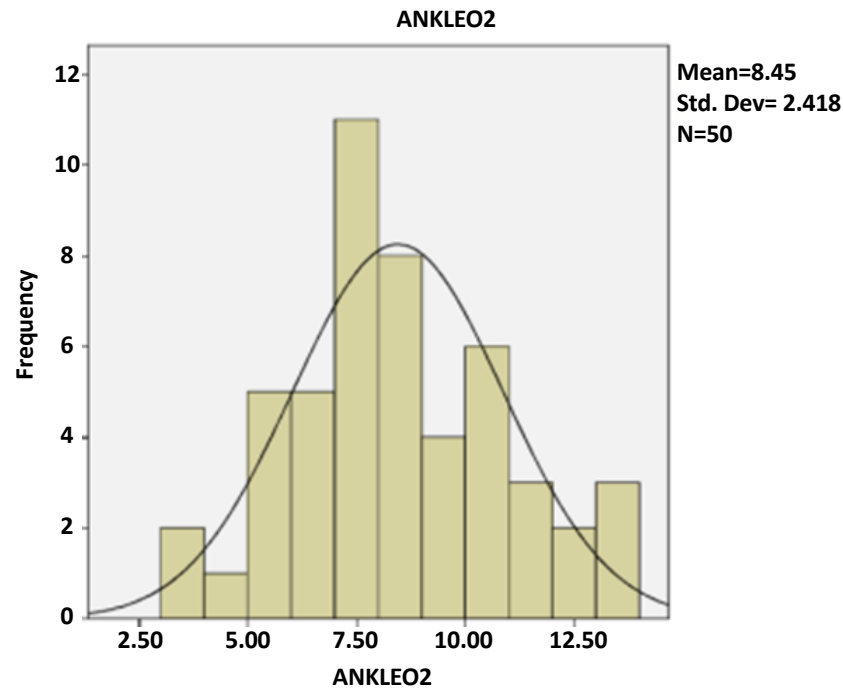

Figure 3. The extent of tibia-fibula distal overlapping in ankles (regardless of right and left and gender)

Orthopedic Science

$55.99 \%$ with $95 \%$ CI in the left ankle and $50.67 \%$ to $56.64 \%$ with $95 \% \mathrm{CI}$ in the right ankle.

The percentage of tibia-fibula distal overlap at $1 \mathrm{~cm}$ from the joint surface ranged from $46.71 \%$ to $54.39 \%$ with a CI of $95 \%$ in females and $50.40 \%$ to $56.28 \%$ with a CI of $95 \%$ in males.

The percentage of tibia-fibula distal overlap at $1 \mathrm{~cm}$ from the joint surface (regardless of side or gender) ranged from $49.43 \%$ to $54.35 \%$ with a CI of $95 \%$ (Figure 4 ).

\section{Discussion}

According to the study results, the range of distal tibiafibula overlap of one centimeter above the tibiotalar joint line (regardless of side or gender) was 7.81 to $9.09 \mathrm{~mm}$ (95\% CI) when the knee is in true AP position. The percentage of tibia-fibula distal overlap at $1 \mathrm{~cm}$ from the joint surface (regardless of side or gender) ranged from $49.43 \%$ to $54.35 \%(95 \% \mathrm{CI})$.

Most tibial diaphysis fractures were treated non-surgically in the past, but surgery has become the most

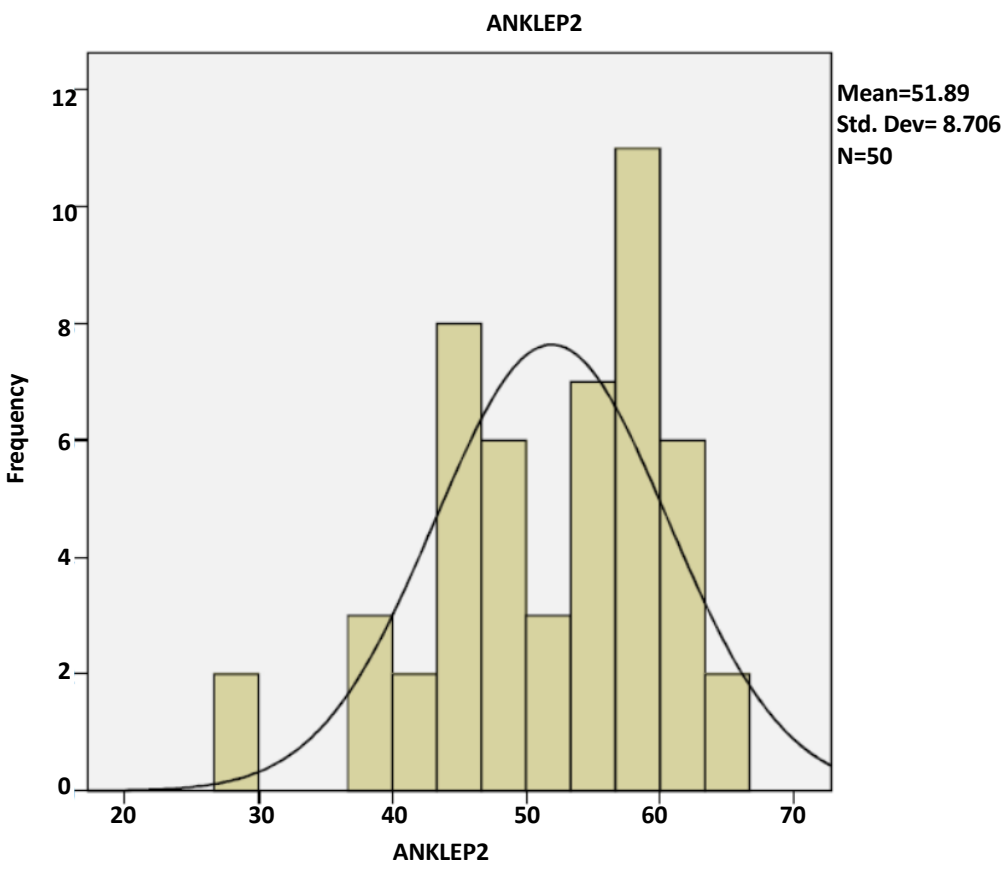

Figure 4. The percentage of tibia-fibula distal overlapping extent in ankles (regardless of right) 
common treatment. The surgical procedure to best treat tibia and fibula bone fractures is the closed fixation with intramedullary nailing [1]. One of the worst complications of this treatment is rotational malalignment. There are different methods of evaluating tibial rotation, such as TFA, TTA, simple radiography [3], ultrasonography [10], fluoroscopy [5, 6], and CT scan.

Among clinical and radiographic methods, a CT scan is the most accurate one for evaluating the distal rotation of the tibia [20]; however, it has many disadvantages, including patient exposure to radiation, its availability and cost.

The extent of tibia-fibula distal overlap will change in AP radiography of an ankle with the rotation in the distal part when the proximal segment is fixed. Radiography of the ankle in this position and considering the extent of the tibiafibula distal overlap can be used to assess tibial malrotation.

The size of the tibia and fibula bones in the measured samples do not have normal distribution curves, so their use in these populations is statistically associated with a high rate of error. As a result, all measurements are presented with a $95 \%$ confidence interval.

Different tissue masses around the ankle may cause some changes in distance and potentially affect the radiographic measurements. Also, the magnitude of magnification may vary with radiology devices and is sometimes associated with high error rates, especially with radiology devices used in operating rooms. Therefore, measuring the percentage of tibia-fibula distal overlap and comparing it with fibula widths at the same level is a much more accurate measurement method.

Staheli et al. [2] and Stulberg et al. [4] developed and improved the Thigh-Foot Angle (TFA) method in 1985 and 1991, respectively, as the easiest clinical method for determining tibial rotation. With this method, the patient is first placed in a prone position. The knee is placed at a 90-degree angle and the ankle joint is placed in neutral flexure. Then, the angle between the foot axis and the femur is equal to the tibial rotation angle. Although this method is the best and easiest clinical method for determining rotational malalignment of tibia and fibula bones, its measured value varies from person to person. In this examination, the patient is in a prone position, which is not possible during operation. It has been shown that the degree of rotational malalignment in the form of External Tibial Torsion (ETT) (up to 22 degrees) and Internal Tibial Torsion (ITT) (up to 31 degrees) has not been identified and overlooked in clinical evaluations [21].
Melih Guven et al. [3] provided another method for determining tibial rotation using radiography. In their method, the maximum distance between internal and external ankles is first measured with AP radiography of the joint surfaces. Then, the distance between internal and external ankles is measured in lateral radiography. Finally, the tangent from the first to the second distance is equal to the tibial rotation. This method has the advantages of being available in all areas, even in the operating room, and exposing patients to less radiation than CT scans.

Its disadvantages include low accuracy in determining the distance between the tibia and the fibula in lateral radiography and its high measurement error, to the extent that the ankle rotation in the lateral position changes the distance between the tibia and the fibula. In this method, two radiographs (AP and lateral) are needed. The method proposed in the current study also requires two radiographs (true AP radiography of the knee and the ankle in the same position), which is performable and available in all environments such as operating rooms. There is no need for mathematical calculations like in the Guven et al. method or true lateral radiography of the ankle, which is sometimes difficult to take.

Clements et al. [6] also introduced a method for determining the amount of tibial rotational malalignment during surgery using fluoroscopy. In this method, a fluoroscopic image is first taken of the lateral knee, and the limb is placed in a position where both femoral condyles are consistent and parallel to the horizontal. In this position, the angle between the line passing through the two malleoli and the horizontal surface would be equal to the tibial rotation. This method is simple and applicable in all environments, especially in the operating room, and exposes the patient to less radiation than with a CT scan. However, it suffers from some disadvantages.

This method is a combination of radiographic and clinical methods because first, it tries to put the lower extremity parallel to a horizontal line; then, the angle between the horizontal and the line passing through both malleoli is measured clinically. Therefore, its measurement error rate is high and can be changed by different measurements. On the other hand, in this method, a true lateral radiograph of the knee is necessary, which is sometimes difficult to obtain. The proposed method is completely radiographic, which can reduce inter- and intra-observer errors.

In this method, it is sometimes necessary to take more than one radiograph from the knee to obtain a true AP image of the knee. However, it is possible to expose the patient 
to less radiation by knowing the position of the knee to take a true AP image and by observing the mentioned standards.

Bonnevialle et al. [21], Gustilo et al. [23], Prasad et al. [8], and Puno et al. [15] have conducted separate studies on clinical and imaging methods for determining tibial rotation and rotational deformity of the tibia and addressed CT scan as the most accurate method. Although this method is the most accurate method for determining rotational malalignment of tibia and fibula bones, it has some disadvantages. It may not be available in all hospitals or operating rooms, and it exposes the patient to too much radiation.

Based on the current study, it is recommended that the percentage of tibia-fibula distal overlap be measured in suspicious cases. If the value is in the normal range, the possibility of malrotation is low. However, if there is any difference, then a comparison with the contralateral side can be helpful. CT scans can be reserved for highly suspicious cases when this method is inconclusive.

The present study also had some limitations. First, the knee radiograph should be in a true AP view, and it may need multiple exposures. To minimize this limitation, it is recommended that the standard method and limb positioning mentioned in the proposed plane procedure be followed. Second, this method can only estimate the presence of tibial malrotation and does not determine the degree of rotational malalignment. Third, this study was done on individuals with normal alignment; it needs to be adjusted for other conditions.

\section{Conclusions}

Based on the results of the current study, distal tibia-fibula overlap when the knee is in the true AP position, regardless of side or gender, is 7.81 to $9.09 \mathrm{~mm}$ or $49.43 \%$ to $54.35 \%$. This finding can be helpful as a measure for determining tibial malrotation during or after operation.

\section{Ethical Considerations}

\section{Compliance with ethical guidelines}

Ethical guidelines of IUMS ethical board were followed in this study.

\section{Funding}

This research did not receive any grant from funding agencies in the public, commercial, or non-profit sectors.

\section{Authors' contributions}

Conceptualization, editing, supervision: Hamidreza Yazdi; Funding acquisition: Hamidreza Yazdi, Mohammad Taher Ghaderi; Investigatng, writing original draft, writing edition: Mohammad Taher Ghaderi; Methodology: Alireza Yousof Gomrokchi; Software, validation: Parham Pezeshk; Resources, data analysis: Parham Pezeshk, Alireza Yousof Gomrokchi.

\section{Conflict of interest}

The authors declared no conflict of interest.

\section{References}

[1] Heckman JD, McQueen MM, Ricci WM, Tornetta P, McKee MD. Rockwood and Green's fractures in adults. Philadelphia: Wolters Kluwer Health; 2015.

[2] Staheli LT, Corbett MA, Wyss CR, King HO. Lower-extremity rotational problems in children. Normal values to guide management. J Bone Joint Surg Am. 1985; 67(1):39-47. [DOI:10.2106/00004623-198567010-00006]

[3] Güven M, Akman B, Ünay K, Özturan EK, Cakıcı H, Eren A. A new radiographic measurement method for evaluation of tibial torsion: A pilot study in adults. Clin Orthop Relat Res. 2009; 467(7):1807-12. [DOI:10.1007/s11999-008-0655-z] [PMID] [PMCID]

[4] Stuberg W, Temme J, Kaplan P, Clarke A, Fuchs R. Measurement of tibial torsion and thigh-foot angle using goniometry and computed tomography. Clin Orthop Relat. 1991; 272:208-12. [DOI:10.1097/00003086-199111000-00031]

[5] Clementz BG. Tibial torsion measured in normal adults. Acta Orthop Suppl. 1988; 59(4):441-2. [DOI:10.3109/17453678809149399] [PMID]

[6] Clementz BG, Magnusson A. Fluoroscopic measurement of tibial torsion in adults. Arch Orthop Trauma Surg. 1989; 108(3):150-3. [DOI:10.1007/BF00934258] [PMID]

[7] Jakob RP, Haertel M, Stussi E. Tibial torsion calculated by computerised tomography and compared to other methods of measurement. J Bone Joint Surg Br. 1980; 62(2):238-42. [DOI:10.1302/0301-620X.62B2.7364840]

[8] Prasad CV, O'sullivan M, Mccarthy P. Ct assessment of torsion following locked im nailing of tibial fractures. J Bone Joint Surg Br. 1998; 80(1S):70.

[9] Puno RM, Vaughan JJ, Stetten ML, Johnson JR. Long-term effects of tibial angular malunion on the knee and ankle joints. J Orthop Trauma. 1991; 5(3):247-54 [DOI:10.1097/00005131199109000-00001] [PMID] 
[10] Joseph B, Carver RA, Bell MJ, Sharrard WJ, Levick RK, Aithal V, et al. Measurement of tibial torsion by ultrasound. J Pediatr Orthop. 1987; 7(3):317-23. [DOI:10.1097/01241398198705000-00014] [PMID]

[11] van der Werken C, Marti RK. Post-traumatic rotational deformity of the lower leg. Injury. 1983; 15(1):38-40. [DOI:10.1016/0020-1383(83)90160-2]

[12] Kenawey M, Liodakis E, Krettek C, Ostermeier S, Horn T, Hankemeier S. Effect of the lower limb rotational alignment on tibiofemoral contact pressure. Knee Surg Sports Traumatol Arthrosc. 2011; 19(11):1851. [DOI:10.1007/s00167-0111482-4] [PMID]

[13] Svoboda MS, McHale CK, Belkoff SM, Cohen KS, Klemme LW. The effects of tibial malrotation on the biomechanics of the tibiotalar joint. Foot Ankle Int. 2002; 23(2):102-6. [DOI:10 .1177/107110070202300204] [PMID]

[14] Turner MS. The association between tibial torsion and knee joint pathology. Clin Orthop Relat Res. 1994; 302:47-51. [DOI:10.1097/00003086-199405000-00009]

[15] Turner MS, Smillie IS. The effect of tibial torsion of the pathology of the knee. J Bone Joint Surg Br. 1981; 63(3):396-8. [DOI:10.1302/0301-620X.63B3.7263753]

[16] Van der Schoot DK, Den Outer AJ, Bode PJ, Obermann WR, Van Vugt AB. Degenerative changes at the knee and ankle related to malunion of tibial fractures. Br J Bone Joint Surg. 1996; 78(5):722-5. [DOI:10.1302/0301-620X.78B5.0780722]

[17] Harper MC, Keller TS. A radiographic evaluation of the tibiofibular syndesmosis. Foot Ankle. 1989; 10(3):156-60. [D OI:10.1177/107110078901000308] [PMID]

[18] Ostrum RF, De Meo P, Subramanian R. A critical analysis of the anterior-posterior radiographic anatomy of the ankle syndesmosis. Foot Ankle Int. 1995; 16(3):128-31. [DOI:10.117 7/107110079501600304] [PMID]

[19] Pneumaticos SG, Noble PC, Chatziioannou SN, Trevino SG. The effects of rotation on radiographic evaluation of the tibiofibular syndesmosis. Foot Ankle Int. 2002; 23(2):107-11. [DOI:10.1177/107110070202300205] [PMID]

[20] Say F, Bülbül M. Findings related to rotational malalignment in tibial fractures treated with reamed intramedullary nailing. Arch Orthop Trauma Surg. 2014; 134(10):1381-6. [DOI:10.1007/s00402-014-2052-2] [PMID]

[21] Bonnevialle P, Andrieu S, Bellumore $Y$, Challé JJ, Rongières M, Mansat M. [Torsional abnormalities and length discrepancies after intramedullary nailing for femoral and tibial diaphyseal fracture. Computerized tomography evaluation of 189 fractures (French)]. Rev Chir Orthop Reparatrice Appar Mot. 1998; 84(5):397-410. [PMID]

[22] Andy C. Knee. Wiki-radiography [Internet]. 2008 [Updated 2008 Mar 14] Available from: http://www.wikiradiography.com/page/Knee

[23] Skaggs DL, Friend L, Alman B, Chambers HG, Schmitz M, Leake $B$, et al. The effect of surgical delay on acute infection following 554 open fractures in children. J Bone Joint Surg. 2005; 87(1):8-12. [DOI:10.2106/00004623-200501000-00003] 
This Page Intentionally Left Blank 